\title{
ASSOCIATION BETWEEN GATA3 AND PATHOLOGIAL AND IMMUNOHISTOCHEMICAL PREDICTIVE AND PROGNOSTIC PARAMETERS IN EARLY BREAST CANCER
}

Priscila M. Souza, Filomena M. Carvalho², Fernando N. Aguiar ${ }^{3}$, Débora Gagliato ${ }^{4}$, Alfredo C. S. D. Barros¹,5

${ }^{1}$ Experimental Physiopatology, Unversidade de São Paulo, School of Medicine - São Paulo (SP), Brazil. ²Department of Pathology, Faculdade de Medicina, Universidade de São Paulo - São Paulo (SP), Brazil. 3 Instituto do Câncer do Estado de Sao Paulo, Faculdade de Medicina, Universidade de São Paulo - São Paulo (SP), Brazil. ${ }^{4}$ Department of Oncology, Hospital Beneficência Portuguesa - São Paulo (SP), Brazil.

${ }^{5}$ Department of Mastology, Hospital Beneficência Portuguesa - São Paulo (SP), Brazil.

Introduction: GATA3 gene, at 10p14, a member of the GATA family with two GATA-type zinc-fingers, encodes the transcription factors GATA - binding protein 3 (GATA3), critical for the luminal breast epithelium development and maintenance. The GATA3 protein is a linear one, with more than 400 aminoacids, that can be recognized by immunohistochemical analysis. Mutations of the GATA3 and loss of the expression of its related protein are implicated in breast cancer development and aggressiveness. As the most frequent transcription factor in luminal tumor cells, GATA3 became an important marker of mammary differentiation in neoplasias of unknown origin, better than mammaglobin and gross cystic disease fluid protein (GCDFP). Objectives: In this study, we aimed at assessing pathological and immunohistochemical variables and their association with GATA3 expression, adding bases for breast carcinogenesis comprehension and BC (Breast Cancer) precision therapy. Methods: GATA3 was analyzed by immunohistochemistry in whole histological sections of tumors from 105 female patients with histological diagnosis of invasive breast carcinoma and at clinical stages I, II and IIIA, who underwent primary surgical treatment (protocol approval number: 1,604,792). GATA3 nuclear expression was determined in percentage of tumor cells and categorized as preserved (positive expression in more than 95\% of cells) or reduced (negative or expression in up to 95\% of tumor cells). GATA3 expression was analyzed according to patient's age, tumor and node pathological stage, histological type, histological and nuclear grade, lymphovascular invasion, estrogen receptor, progesterone receptor, androgen receptor, HER2 status, and Ki-67. Results: GATA3 expression was detected in 103/105 (98.1\%) cases. Reduced expression was associated with higher histological and nuclear grade, negative hormonal receptors, HER2-positive and higher proliferative activity according to Ki-67 expression. Triple negative breast carcinomas (TNBC) and ER-negative/HER2-positive presented the highest frequency of GATA3 reduction (75\%) compared to ER-positive/HER2-negative (4.1\%) and ER-positive/HER2-positive (20\%). Proliferative activity in TNBC tended to be higher among tumors with GATA3 reduced, irrespective of androgen receptor expression. In the group of ER-positive/ HER2-negative tumors only 3 cases presented GATA3 reduction, all of them with high proliferative activity. Conclusions: GATA3 expression is present in almost all cases of early breast cancer. Reduction in its expression is associated with adverse prognostic factors and higher proliferative activity in all subtypes, including ER-positive/HER2-negative tumors. 